\title{
Iterg - Un nouvel outil de raffinage
}

Oléagineux, Corps Gras, Lipides. Volume 10, Numéro 1, 11-4, Janvier - Février 2003, La filière, aujourd'hui, demain

Auteur(s) : Anne ROSSIGNOL-CASTERA, Institut des Corps Gras, ITERG, rue Monge, Parc Industriel, 33600 Pessac.

Résumé : Le raffinage constitue une étape clef de la technologie de production des huiles et corps gras naturels d'origine végétale ou animale, permettant d'obtenir une qualité conforme aux exigences des différents secteurs utilisateurs : alimentation humaine, alimentation animale, cosmétique, pharmacie, lipochimie. Le raffinage permet en effet d'obtenir conjointement une standardisation de la qualité marchande et organoleptique avec l'obtention d'huiles neutres, non peroxydées, peu colorées et désodorisées, et une garantie d'innocuité avec l'élimination efficace des éventuels contaminants chimiques (solvants organiques, métaux lourds, hydrocarbures polycycliques, résidus phytosanitaires, dioxines, toxines...) et microbiologiques pouvant être présents dans les corps gras bruts. Sous réserve d'être conduit dans des conditions opératoires maîtrisées et adaptées aux caractéristiques de chaque corps gras traité, le raffinage apparaît comme un moyen efficace pour proposer aux consommateurs ou aux industriels, des matières premières naturelles répondant aux obligations de conformité et de sécurité telles que définies dans le Code de la Consommation. Soumis à un encadrement réglementaire précisant les opérations et les produits autorisés (notamment le Décret $n^{\circ}$ 73-139 et les arrêtés du 12 février 1973), le raffinage reste cependant une technologie dépendant bien sûr du savoir faire et de bonnes pratiques de conduite mais aussi, d'une connaissance suffisante des propriétés physico-chimiques spécifiques et de la stabilité des corps gras glycéridiques. Cette double maîtrise est indispensable pour adapter à chaque corps gras brut les paramètres opératoires qui permettront d'éliminer efficacement les composés indésirables sans dégrader ou perdre les constituants natifs intéressants tels que les acides gras polyinsaturés, les stérols ou la vitamine E. L'ITERG, en tant que CentreTechnique Industriel, réunit cette double compétence technologique et scientifique et apporte l'environnement et l'expérience nécessaires pour développer, avec les industriels et pour les industriels, des travaux d'optimisation et d'innovation de cette technologie. Pour réaliser ces travaux, il a été choisi un équipement de raffinage totalement modulaire, assurant la fiabilité et la reproductibilité des paramètres opératoires et permettant la traçabilité du produit à chaque étape du raffinage chimique ou physique : neutralisation, démucilagination, décoloration, décirage, désodorisation et ceci à une échelle représentative d'un process semi-industriel.

\section{ARTICLE}

Contexte de la création de l'outil

Le raffinage constitue une étape clef de la technologie de production des huiles et corps gras naturels d'origine végétale ou animale, permettant d'obtenir une qualité 
conforme aux exigences des différents secteurs utilisateurs : alimentation humaine, alimentation animale, cosmétique, pharmacie, lipochimie. Le raffinage permet en effet d'obtenir conjointement une standardisation de la qualité marchande et organoleptique avec l'obtention d'huiles neutres, non peroxydées, peu colorées et désodorisées, et une garantie d'innocuité avec l'élimination efficace des éventuels contaminants chimiques (solvants organiques, métaux lourds, hydrocarbures polycycliques, résidus phytosanitaires, dioxines, toxines...) et microbiologiques pouvant être présents dans les corps gras bruts. Sous réserve d'être conduit dans des conditions opératoires maîtrisées et adaptées aux caractéristiques de chaque corps gras traité, le raffinage apparaît comme un moyen efficace pour proposer aux consommateurs ou aux industriels, des matières premières naturelles répondant aux obligations de conformité et de sécurité telles que définies dans le code de la Consommation. Soumis à un encadrement réglementaire précisant les opérations et les produits autorisés (notamment le Décret $n^{\circ}$ 73-139 et les arrêtés du 12 février 1973), le raffinage reste cependant une technologie dépendant bien sûr du savoir faire et de bonnes pratiques de conduite mais aussi, d'une connaissance suffisante des propriétés physico-chimiques spécifiques et de la stabilité des corps gras glycéridiques. Cette double maîtrise est indispensable pour adapter à chaque corps gras brut les paramètres opératoires qui permettront d'éliminer efficacement les composés indésirables sans dégrader ou perdre les constituants natifs intéressants tels que les acides gras poly-insaturés, les stérols ou la vitamine E. L'ITERG, en tant que CentreTechnique Industriel, réunit cette double compétence technologique et scientifique et apporte l'environnement et l'expérience nécessaires pour développer, avec les industriels et pour les industriels, des travaux d'optimisation et d'innovation de cette technologie. Pour réaliser ces travaux, il a été choisi un équipement de raffinage totalement modulaire, assurant la fiabilité et la reproductibilité des paramètres opératoires et permettant la traçabilité du produit à chaque étape du raffinage chimique ou physique: neutralisation, démucilagination, décoloration, décirage, désodorisation et ceci à une échelle représentative d'un process semiindustriel.

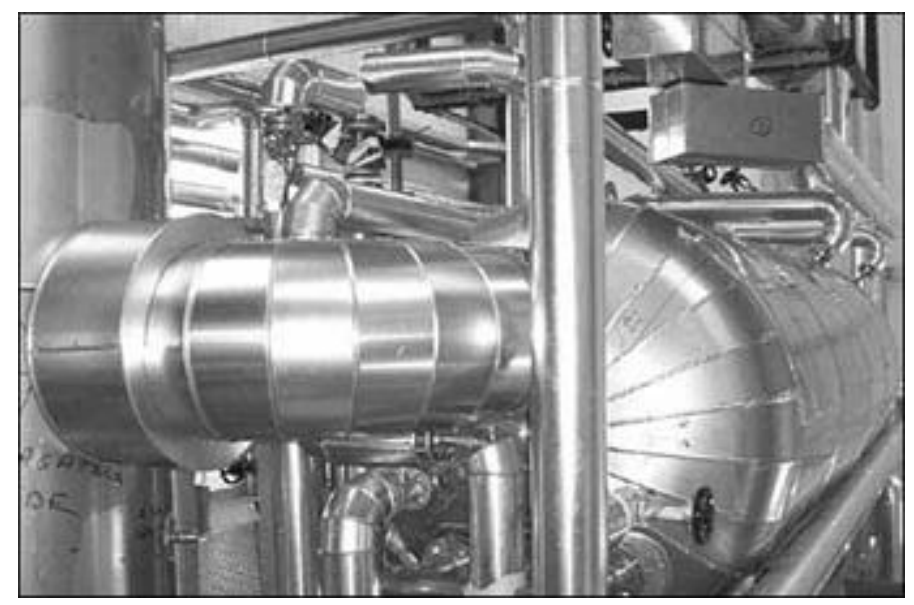


Les finalités de l'outil

La ligne de raffinage de l'ITERG est adaptée pour réaliser d'une part des travaux de Recherche et de Développement et d'autre part des productions à façon de lots d'huiles raffinées. Dès 2003, il est prévu un programme d'optimisation de la désodorisation neutralisante des huiles insaturées en vue de limiter la formation $\mathrm{d}$ 'isomères trans des acides gras et la dégradation des tocophérols. L'efficacité comparée du décirage par filtration ou centrifugation sera également étudiée. Dans le cadre de différents programmes de recherche nationaux et européens sur la valorisation alimentaire et non alimentaire des coproduits générés par le raffinage, seront obtenus des échantillons représentatifs de différents mucilages, pâtes de neutralisation, terres de décirage ou de décoloration, déo-distillats, afin de les caractériser et d'en tester les fonctionnalités pour des usages lipochimiques ou cosmétiques. Dans le contexte général actuel de sécurité alimentaire, sera étudié l'impact du procédé de raffinage sur l'élimination de certains contaminants chimiques tels que les pesticides ou les dioxines, sur le devenir des traces de protéines et d'ADN dans le cas du traitement d'huiles issues de graines OGM ou de graines à risque allergène. Dans le domaine de la qualité marchande des huiles, sera étudié le suivi au cours du raffinage des composés d'oxydation non volatils autres que les peroxydes (aldéhydes, acides oxydés...) pouvant être des marqueurs de la qualité des graines ou des fruits d'origine. Dans le cas des huiles biologiques, cet outil sera particulièrement utile pour développer un "soft-raffinage"compatible avec le label biologique et permettant d'obtenir des huiles désodorisées, peu oxydées et exemptes de composés indésirables tels que les aflatoxines ou les protéines allergènes (cas de l'arachide et du sésame par exemple). Pour réaliser des fabrications en sous-traitance, ce pilote est particulièrement adapté pour traiter soit des corps gras bruts fluides ou concrets difficiles à raffiner car très acides, colorés (beurres exotiques, graisses animales ...) ou riches en phospholipides ou en insaponifiable, soit des huiles fragiles polyinsaturées telles que les huiles de poisson ou les huiles linoléniques (chanvre, lin, noix, onagre, bourrache...). II n'existait pas d'outil équivalent à ce jour en Europe permettant d'offrir simultanément des garanties de performance et de traçabilité du matériel et de compétence des opérateurs, au c?lur d'un environnement technique et scientifique adapté, et disponible pour de petites productions d'huiles raffinées de bonne qualité. Or certains secteurs tels que la cosmétique, la nutraceutique, la pharmacie et la chimie fine sont demandeurs de tonnages réduits pour des huiles particulières, souvent non métropolitaines, pour lesquelles le raffinage n'est pas toujours maîtrisé. Enfin, nous pouvons noter que ce nouvel outil permettra de former ou de perfectionner des ingénieurs ou des techniciens dans des conditions représentatives des procédés industriels. 


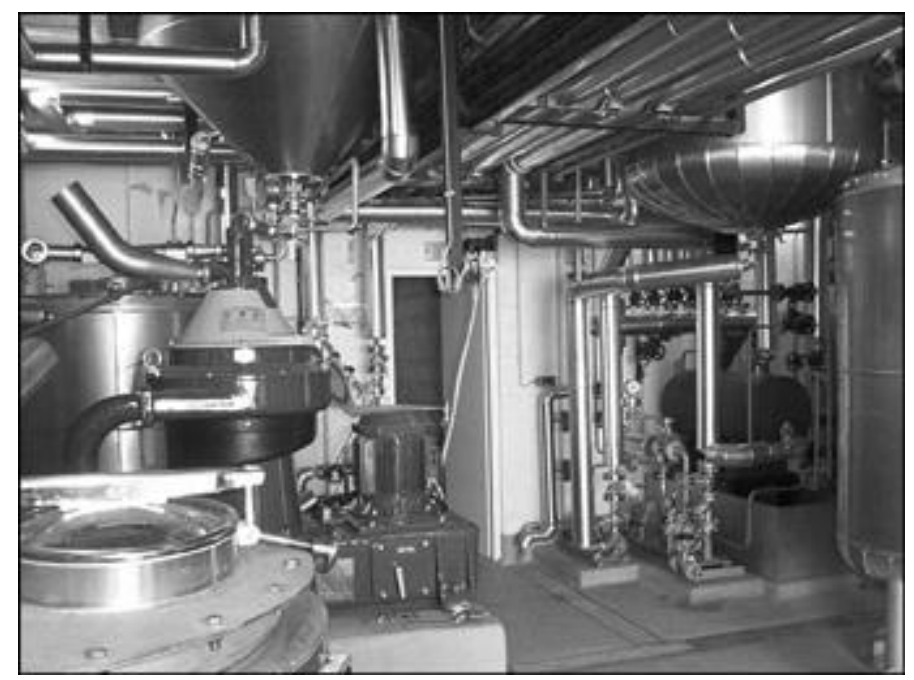

Les utilisateurs potentiels et leurs attentes

L'équipement de raffinage de I'ITERG peut répondre aux besoins des industriels producteurs de corps gras et à ceux des industriels utilisateurs ou metteurs en marché de corps gras. Selon leur nature, les travaux demandés peuvent être réalisés soit dans le cadre collectif et public du programme annuel de recherche de l'ITERG, soit dans un cadre contractuel bipartite avec des garanties de stricte confidentialité et de traçabilité conformes aux contraintes industrielles. Lors d'une enquête réalisée en octobre 2000 auprès de 30 entreprises françaises et de 40 entreprises européennes (Italie, Espagne, Grande-Bretagne, Benelux, Allemagne) représentatives des secteurs producteurs, distributeurs et utilisateurs de corps gras, $50 \%$ des interlocuteurs ont manifesté un intérêt pour l'existence d'une plate-forme de raffinage en France et $20 \%$ se déclaraient utilisateurs potentiels à court terme. Parmi ceux-ci, nous pouvons citer les producteurs et distributeurs d'huiles végétales pour la cosmétique et la nutraceutique et les PME productrices d'huiles biologiques. Le potentiel de production d'une tonne d'huile raffinée pour 2 à 3 jours de fonctionnement, répond en majorité aux besoins exprimés. Qualitativement, les utilisateurs attendent des garanties de traçabilité, de compétence, de confidentialité et de neutralité. Ils sont par ailleurs sensibles à la possibilité d'intégrer l'obtention de I'huile brute par pression ou par extraction hexane à partir de graines ou de fruits, sur le même site et avec des quantités de production équivalentes, en utilisant les équipements de trituration des ateliers de CREOL*, structure expérimentale filiale du CETIOM* et de SOFIPROTEOL*. Parmi les attentes exprimées par les huiliers triturateurs, raffineurs ou conditionneurs, il est cité l'optimisation des procédés (décirage, désodorisation), la validation de l'impact du raffinage sur la qualité des produits (résidus phytosanitaires, protéines) et la production d'échantillons dans des conditions contrôlées ou de premiers lots d'une nouvelle huile. Les producteurs d'huiles vierges ou biologiques souhaitent disposer d'un outil de désodorisation performant et espèrent des perspectives d'innovation en matière de "soft-raffinage". 
Pour les distributeurs d'huiles et les industriels de la nutrition et de la cosmétique, cet équipement de raffinage est une alternative prometteuse pour produire à la demande de leurs clients, des petits tonnages d'huiles à forte valeur ajoutée, dont des huiles exotiques souvent issues de filières de production aléatoires au niveau quantitatif et qualitatif. Les secteurs de l'alimentation animale ou de la lipochimie souhaitent utiliser le pilote pour produire et tester différents coproduits d'intérêt nutritionnel ou technologique: huiles acides, cires, stérols, etc., en vue de leur valorisation.

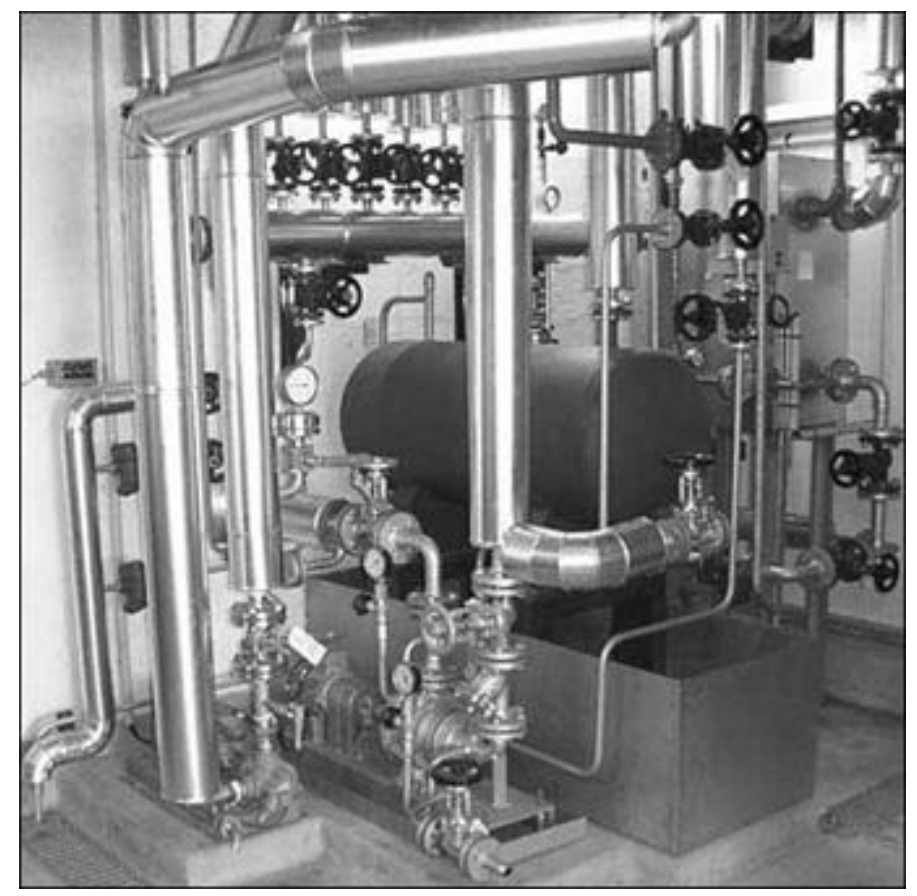

Description de l'équipement

Les étapes du raffinage des corps gras sont données dans le schéma ci-après mentionnant les spécificités propres à la ligne de l'ITERG.

Les critères principaux suivants ont été retenus au niveau de la conception de cette ligne: équipement discontinu adapté pour le raffinage de lots d'huiles de composition et de qualité différentes, souplesse de l'installation au niveau de la capacité de traitement (batch unitaire de 300 à $900 \mathrm{~kg}$ d'huile), équipements performants adaptés pour traiter dans de bonnes conditions des huiles fragiles ou jugées difficiles à raffiner, chauffage rapide dans les réacteurs, maîtrise des paramètres opératoires en conformité avec un système qualité du type ISO 9001 (capteurs, régulation adaptée, acquisition informatique de données, supervision du process), nettoyage de l'installation efficace. La ligne de raffinage, conçue et fabriquée par la société belge "Crystallisation \& Degumming" comprend deux réacteurs inox à double enveloppe, d'une capacité individuelle de $1000 \mathrm{~kg}$ d'huile, le premier dédié aux opérations de démucilagination à l'eau ou à l'acide, de 
neutralisation chimique et aux lavages, le deuxième dédié à la décoloration sous vide (température maximale de $150^{\circ} \mathrm{C}$ ) ou au décirage (température minimale de $6{ }^{\circ} \mathrm{C}$ ). Ces réacteurs sont dotés d'équipements complémentaires: filtre presse, filtre de polissage, centrifugeuse auto-débourbeuse, pompes et bacs tampon. L'équipement de désodorisation, point essentiel et critique d'une ligne de raffinage, est particulièrement performant. II s'agit d'un désodorisateur horizontal à couche mince à deux compartiments permettant un traitement jusqu'à $260^{\circ} \mathrm{C}$ et un vide de 3 millibars. La conception de ce matériel permet une grande efficacité d'élimination des composés volatils, une faible consommation de vapeur de stripping minimisant le phénomène d'hydrolyse, une souplesse réelle au niveau de la capacité (380 à $820 \mathrm{~kg}$ pour 1 ou 2 compartiments), un refroidissement efficace dans le désodorisateur et la possibilité de travailler avec des temps de séjour réduits. En sortie de désodorisateur, I'huile peut être anti-oxydée en ligne et inertée à l'azote. L'ensemble de l'installation est calorifugée et tracée afin de raffiner des graisses à haut point de fusion sans risque de cristallisation dans les tuyauteries. L'installation tout inox est prévue pour effectuer un nettoyage en place et une purge optimale des capacités. Un prélèvement d'échantillon est possible en cours de fonctionnement au niveau de chaque réacteur et capacité. Des aires spécifiques ont été prévues pour le stockage des matières premières et des produits finis: chambre froide pour les huiles fragiles et chambre chaude jusqu'à $90^{\circ} \mathrm{C}$ pour les corps gras concrets. L'atelier pilote intègre un poste de contrôle analytique pour les suivis de base au cours du raffinage ; tous les autres contrôles analytiques (peroxydes, phosphore, savons, couleur, acides gras, tocophérols...) pouvant être réalisés sur place dans les laboratoires accrédités NF-EN-ISO/CEI 17025 de l'ITERG.

CARACTERISTIQUES DE L'ATELIER PILOTE DE RAFFINAGE

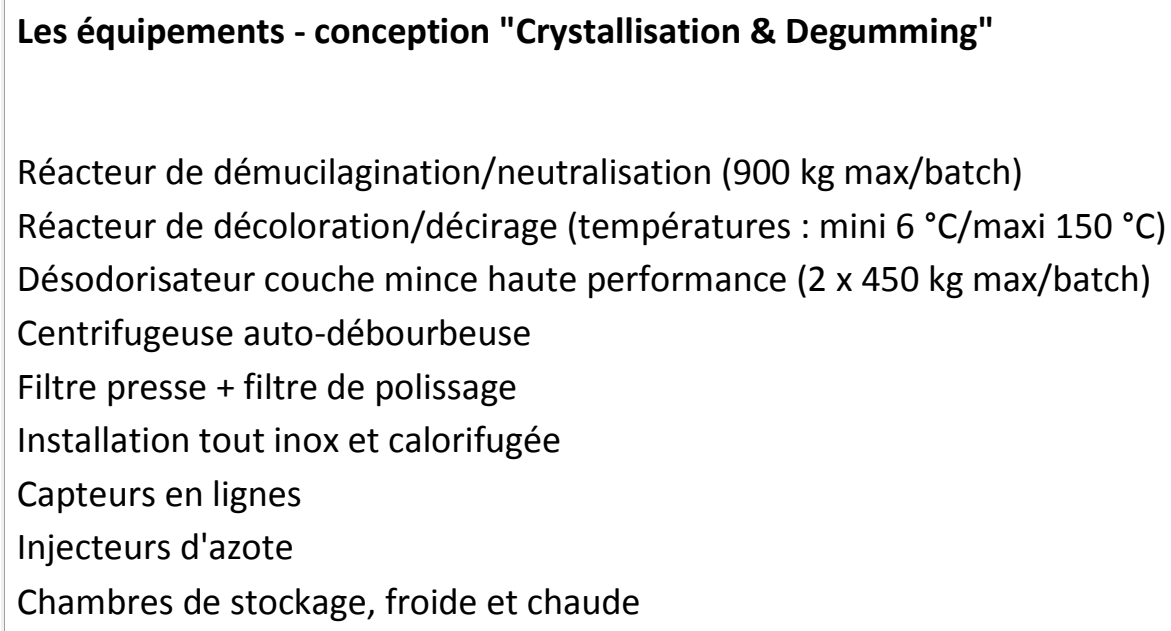




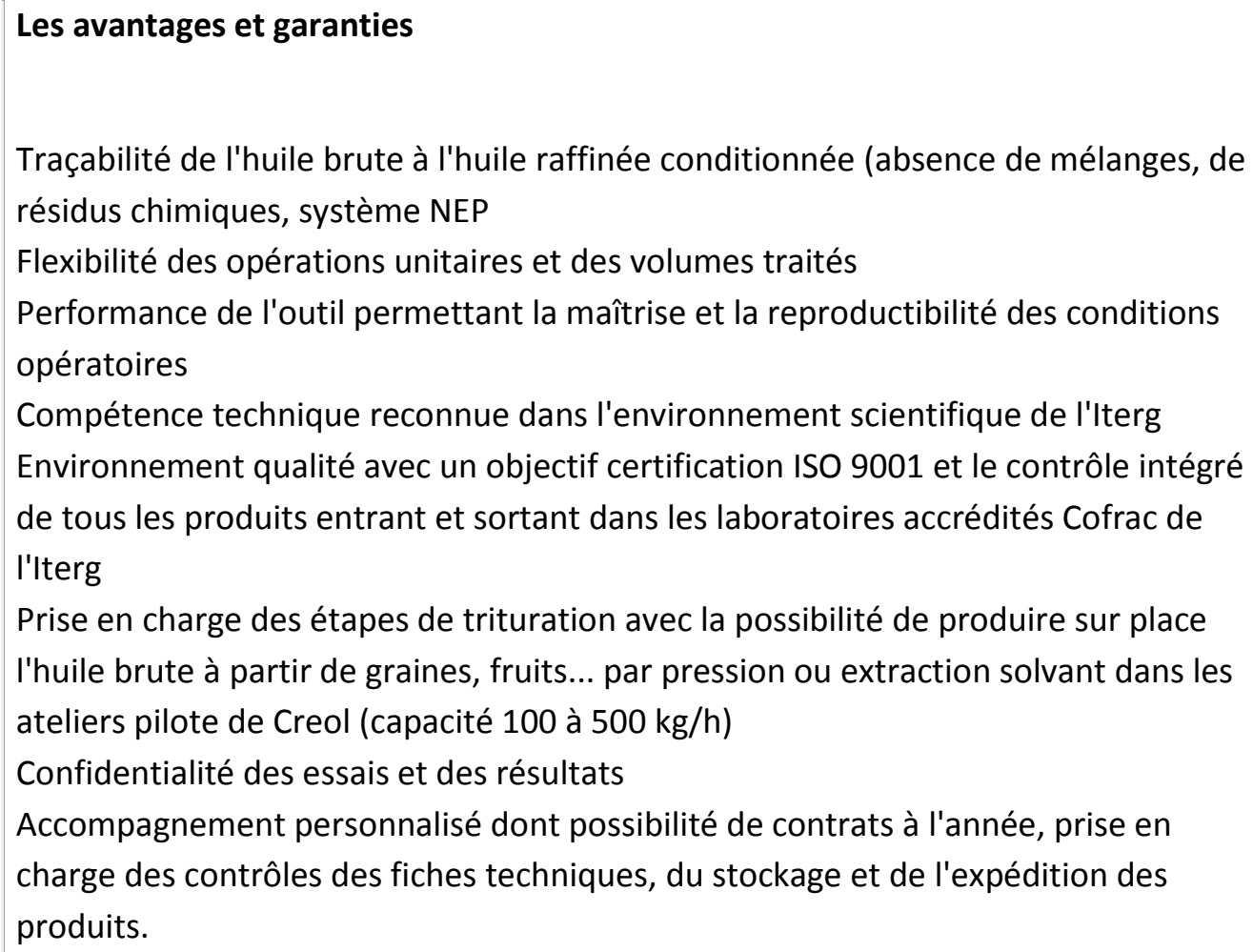

\section{Conclusion}

Avec une ligne de raffinage performante et répondant aux exigences de qualité et de traçabilité, l'ITERG offre aux industriels producteurs et utilisateurs d'huiles végétales et de corps gras naturels, un outil de Recherche et de Production permettant de contribuer efficacement au développement de nouvelles huiles et de nouvelles applications.

Cet outil permet de raffiner partiellement ou totalement par voie chimique ou physique, tout corps gras quelque soit sa qualité et sa composition, avec des batchs de production de $300 \mathrm{~kg}$ à plusieurs tonnes, une garantie sur les conditions opératoires mises en ?uvre, des performances technologiques optimales en particulier pour l'étape de désodorisation et un suivi analytique complet des produits obtenus. Avec un environnement unique en Europe au sein d'une structure pluridisciplinaire** spécialisée sur les corps gras, ce pilote bénéficie de moyens humains et scientifiques permettant d'optimiser et de personnaliser chaque demande dans un contexte de confidentialité et de neutralité. Opérationnel à compter de janvier 2003, gageons que ce nouvel outil sera largement utilisé par les professionnels de la filière et par les industriels utilisateurs de corps gras raffinés. 


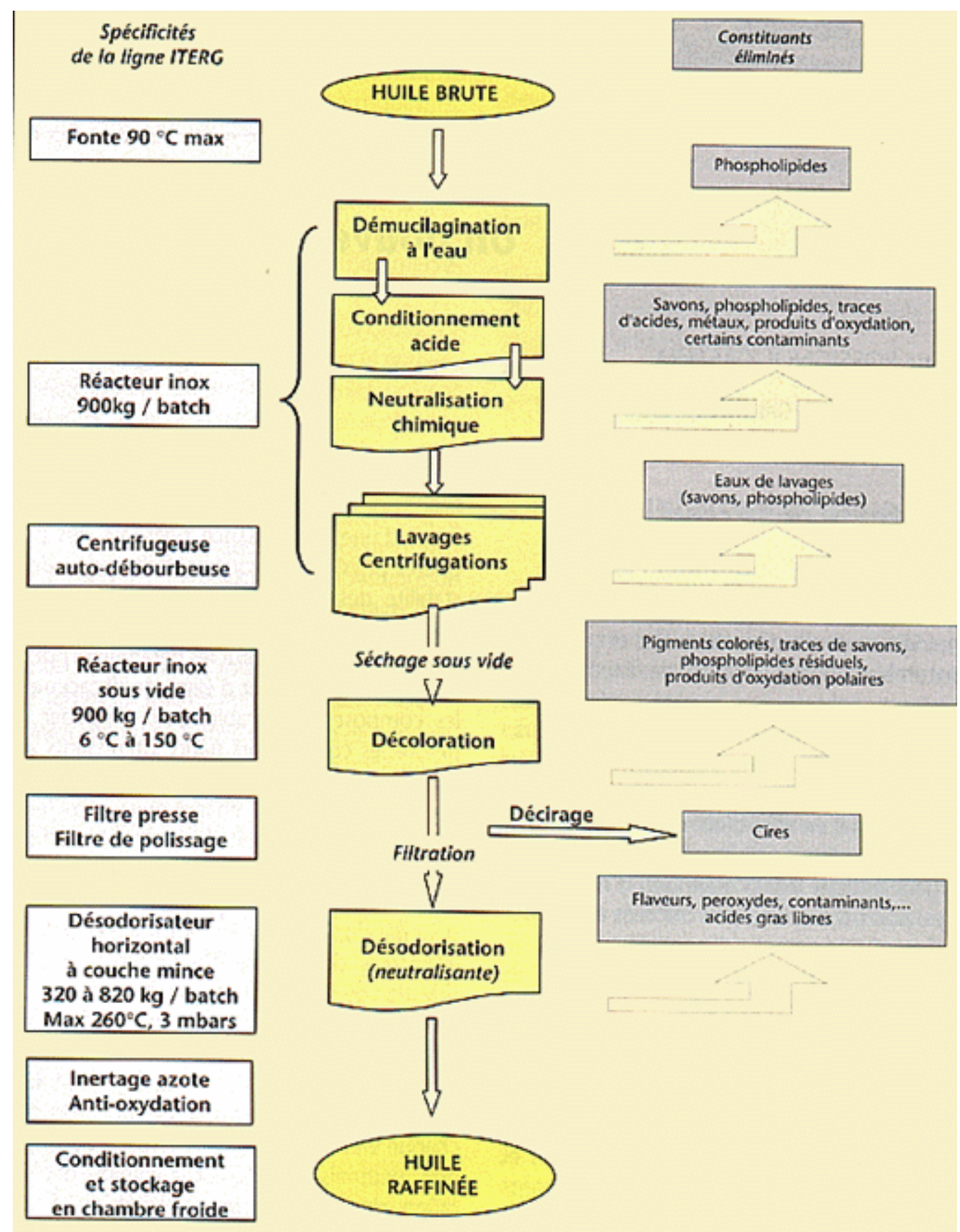

- Étapes du raffinage des corps gras et spécificités propres à la ligne de l'ITERG

\section{Contacts}

Xavier PAGES, Responsable Technologie (33) 0556079772 - x.pages@iterg.com

Armelle JUDDE, Marketing Développement (33) 0556079773 - a.judde@iterg.com

* CREOL : Centre de Recherche et d'Expérimentation sur les Oléagineux

CETIOM : Centre Technique Interprofessionnel des Oléagineux Métropolitains 
SOFIPROTEOL : Etablissement financier de la filière française des huiles et protéines végétales

** L'ITERG comprend 8 départements opérationnels: Prestations Analytiques,

Recherche Analytique, Biochimie-Nutrition, Chimie-Rhéologie, TechnologieEnvironnement, Documentation, Audits \& Conseil, Formation. 